\title{
Preocupación y deterioro de la calidad ambiental. Apreciación de los estudiantes universitarios*
}

Pedro César Cantú-Martinez ${ }^{\mathrm{a}}$

Universidad de Nuevo León, México

cantup@hotmail.com

ORCID: https://orcid.org/0000-0001-8924-5343
DOI: https://doi.org/10.11144/Javeriana.ayd24-46.pdca

Recibido: 10 Noviembre 2019

Aceptado: 15 Febrero 2020

Publicado: 30 Junio 2020

\section{Resumen:}

En el presente trabajo, se aborda el pensamiento y la manera de interiorizar el vínculo que existe entre el individuo y el ambiente en el que vive. El estudio se realizó en la Universidad Autónoma de Nuevo León (México), con la participación de 65 estudiantes universitarios. Como metodología, se empleó la Encuesta Internacional sobre Actitudes y Acciones de los Estudiantes Europeos con Relación a Temas Ambientales, Sección Problemas Ambientales, y esta fue valorada mediante la escala de Likert. La población promediaba una edad de 19,3 años, $41,5 \%$ fueron mujeres y $58,4 \%$ varones; de estos, $57 \%$ están preocupados y $43 \%$ poco preocupados. Las tres problemáticas principales que se identificaron fueron las industrias y pedreras, el tráfico y el ruido. Se concluye, además, que hay una similitud de opiniones del $39,1 \%$ entre mujeres y varones, y se confirma que las mujeres presentan una mayor preocupación que los varones.

Palabras clave: percepción, conocimiento, ambiente, calidad ambiental, estudiantes universitarios.

Abstract:

This work addresses how college students think and internalize the link between the individual and the environment he/she lives in. This study was conducted at the Universidad Autónoma de Nuevo León (Mexico) and 65 college students took part in it. The methodology consisted in an international survey about European Student's Attitudes and Actions regarding Environmental Topics, in the Section of Environmental Problems. The survey was answered using a Likert scale. The population age average was 19.3 years, $41.5 \%$ were women and $58.4 \%$ men. Out of them, $57 \%$ are worried and $43 \%$ are a little worried. The three main problems identified were the industries and stone quarries, the traffic, and the noise. It is also concluded that $39.1 \%$ of female and male students had similar opinions and it is confirmed that women are more worried than men.

Keywords: perception, knowledge, environment, environment quality, college studentIntroducción.

La preocupación ambiental ha retomado suma importancia en el mundo, especialmente, debido al detrimento creciente de las particularidades esenciales que sustentan a nuestra sociedad,. por ejemplo, los efectos negativos de la disminución en la calidad ambiental. De acuerdo con Enrique-Mirón et al. (2012), algunas eventualidades ambientales progresan rápidamente como "nuevas enfermedades, extinción de especies, desertización, hambrunas, contaminación de las aguas, destrucción del patrimonio cultural, agotamiento de los recursos, contaminación atmosférica, etc., quedando asimismo claro que la velocidad y dimensiones adquiridas eran muy superiores a las predichas" (p. 2).

En efecto, los sucesos ambientales que hemos mencionado anteriormente exteriorizan el comportamiento irracional del ser humano, en el que prevalecen las determinaciones de llevar a cabo adecuaciones en el ambiente y, en cambio, lo único que se ha conseguido es la alteración de lo que ya desde antes se encontraba en equilibrio en el entorno natural (Clavijo et al., 2018). Es así que "las diversas actividades que para sobrevivir han desarrollado las poblaciones humanas, han modificado la estructura y funcionamiento de los diferentes ecosistemas” (Marín-Muñiz et al., 2016, p. 54).

Notas de autor 
Ante este escenario, resultan muy pertinentes los estudios sobre las precepciones, ya que, según Calixto y Herrera (2010), las

percepciones ambientales aportan la información esencial para determinar las ideas que el sujeto se forma del medio ambiente, así como sus actitudes hacia él, es a partir de las ideas y conocimientos. En este sentido radica la importancia de la investigación sobre las percepciones ambientales para la educación ambiental. (p. 246)

Con sustento en lo anterior, debemos considerar que los problemas ambientales cuentan con distintos factores que los posibilitan, los cuales pueden ser "ecológicos, sociales, económicos, culturales, políticos, éticos, etc., a los que nos referimos habitualmente con expresiones como causalidad múltiple o complejidad multicausal, resultando difícil seleccionar cuáles son las causas más importantes" (Álvarez y Vega, 2009, p. 252).

Esto implica un gran desafío para la sociedad, por lo que uno de los propósitos de este escrito es analizar el nivel de pensamiento y reflexión que tienen los estudiantes universitarios del área de ciencias biológicas desde una perspectiva colectiva y de género, con un sustento empírico-analítico, lo que nos permite explorar el vínculo existente entre el individuo y su ambiente, y, además, cómo el estudiante interpreta este constructo.

En suma, se busca plantear una aproximación a la comprensión de la información que poseen los estudiantes, el campo de representación que erigen en función de esta y la actitud que surge ante la realidad que se yergue frente a ellos (Calixto, 2009).

\section{Marco conceptual}

Desde hace ya tiempo, el impulso que han tenido los trabajos de investigación en el ámbito de la percepción ha permitido identificar el creciente interés que esta ha cobrado en los distintos campos del saber, como lo hacía evidente Vargas en 1994. Este mismo autor señala que la "percepción es biocultural porque, por un lado, depende de los estímulos físicos y sensaciones involucrados y, por otro lado, de la selección y organización de dichos estímulos y sensaciones" (p. 47).

Es así que podemos encontrar distintas aproximaciones referentes a la conceptualización de la percepción, entre ellas, la citada por Carterette y Friedman en 1982, quienes plantean que se trata de un elemento constituyente de la conciencia humana que se ostenta en los hechos y que se yergue por la realidad que una persona experimenta. Mientras, Barthey (1982) en su obra Principios de percepción aduce que esta es llanamente el mecanismo por el cual todo ser humano forma conocimiento de las situaciones que lo rodea, además, agrega que se constituye en una comprensión que surge inmediatamente, mediante la cual podemos elaborar un discernimiento o emitir una opinión.

En conexión con lo anterior, Arias (2006) comenta que

a) los estudios de percepción de personas y percepción social han estado muy ligados a los estudios de percepción de objetos. Esencialmente, se parecen en lo siguiente:

Las percepciones están estructuradas, las percepciones no constituyen un continuo procesamiento de estímulos caóticos que se van almacenando, sino que cuando se perciben personas y objetos se crea un orden. Una de esas formas de organización es crear categorías, en el mundo de los objetos físicos las categorías son claras. En la percepción de personas y sus acciones se posee multitud de categorías para clasificar su conducta, su apariencia, y demás elementos informativos: puede ser categorizadas desde diferentes aspectos. Las personas difieren según las categorías que utilizan para categorizar. Hay personas que utilizan un solo sistema categorial, mientras que otras utilizan un sistema más complejo de categorización. 
b) Tanto en la percepción de objetos como de personas se tiende a buscar elementos invariantes de los estímulos que se perciben. Siendo el interés predecir la conducta de los demás, no son interesantes aquellos aspectos de su conducta que parecen superficiales e inestables.

c) Las percepciones de los objetos y de los demás tienen significado. Los diversos estímulos que se perciben pasan al interior de la mente a través de un tamiz. Cuya función primordial consiste en "interpretar" otorgándoles significado. (p. 11)

En este sentido, las percepciones no son consideradas como sucesos aislados, sino que estos se producen de manera continua, como un producto de la interacción entre el sistema natural y el social que constituyen el ambiente. Por lo tanto, las percepciones, de manera general, permiten a todo ser humano construir para sí un contexto de la realidad, tanto en espacio como en tiempo; este constructo es captado por los sentidos y permite generar en las personas una serie de sensaciones que permean de manera consciente o inconsciente, lo que les permite conjeturar frente a lo que los rodea (Spinzi et al., 2017). De hecho, Calixto y Herrera (2010) exponen que estas percepciones

no se encuentran aisladas, intervienen diversas características, con las cuales el sujeto se encuentra conviviendo en su cotidianidad, y percibe a través de los sentidos, lo que otros no alcanzan a percibir, por lo que es común ver o escuchar lo que de forma emocional queremos o para lo que estamos preparados, dado que la percepción no puede deslindarse de la personalidad, así el perceptor interpreta dependiendo de las circunstancias que vive y experimenta. (p. 229)

De esta manera, "el análisis reflexivo a partir de nuestra experiencia del mundo se remonta al sujeto como a una condición de posibilidad distinta del mismo y hace ver la síntesis universal como algo sin lo cual no habría mundo" (Merleau-Ponty, 1993, p. 9). Por ello, la percepción desencadena juicios de valor, conductas y actuaciones que desembocan en acciones, con unos contenidos de orden consecuencial, tanto positivos como negativos (Pidgeon, 1998); en consecuencia, las personas se hacen "sensibles a las condiciones ambientales que les rodean, reconocen mejor las formas normalmente imperceptibles en que el ambiente afecta las actividades humanas" (Calixto y Herrera, 2010, p. 232).

Entonces, ¿ por qué son importantes los estudios relativos a las precepciones ambientales? Porque, tal como lo expone Fernández (2008), por lo general, el contexto de la realidad expresa solo las configuraciones y, en muchos casos, los intereses de las personas que toman las decisiones; por lo tanto, estas personas sobreponen sus apreciaciones por encima de las observables por el colectivo social, lo que tiene como resultado una desarticulación en las valoraciones y una descontextualización de lo que sucede en la realidad. En especial, "el estudio de las percepciones ambientales ayuda a comprender de una mejor manera la relación que existe entre el ser humano y el ambiente, ya que esta relación está determinada por la forma en que se percibe el entorno" (Fernández, 2008, p. 194). La indagación sobre las percepciones es de suma relevancia, ya que, como mencionaba Edgardo Lander en 1984 (citado por Calixto, 2009), la problemática ambiental y sus consecuencias "han pasado a formar parte de la conciencia colectiva de amplios sectores de la humanidad y ha incidido en términos prácticos en cambios de conducta significativos y de actitud en relación al ambiente" ( $p$. 23).

Con base en lo anterior, en este trabajo se intenta, mediante la percepción, aprehender la relación que subsiste entre el ámbito cognitivo y los valores que surgen del espacio subjetivo, en el que se desarrolla una dialéctica entre el contexto natural y el social, y el ser humano, en la que se distingue "lo simbólico, lo afectivo, lo vivido y lo subjetivo, que se denomina espacio percibido" (Padilla y Luna, 2003, p. 103). Lo anterior es consecuencia de las actividades cotidianas que no permiten una correcta percepción, o bien, se rinde la existencia a una normatividad social como ética del comportamiento. 


\section{Metodología}

El presente estudio es de carácter transversal, descriptivo y comparativo, y fue posible mediante la realización de un muestreo por conveniencia, en el que se encuestaron a 65 estudiantes de la carrera de Químico Bacteriólogo Parasitólogo, que cursaban tercer semestre, del área de ciencias biológicas, y se llevó a cabo entre agosto y octubre de 2019, en la Universidad Autónoma de Nuevo León (UANL).

Para la obtención de los resultados, se utilizó el instrumento de Oliver et al. (2005), denominado Encuesta Internacional sobre Actitudes y Acciones de los Estudiantes Europeos con Relación a Temas Ambientales-Sección Problemas Ambientales. La finalidad era establecer una relación entre el instrumento y las condiciones ambientales del Área Metropolitana de Monterrey (AMM), N.L., México. La ANM cuenta con una población cercana a los 4,7 millones de habitantes y está integrada por 13 municipalidades que albergan el $88 \%$ de la población total del Estado de Nuevo León.

La evaluación de los resultados se logró a partir de una escala de Likert, con valores de 0-3. Esto permitió valorar la gravedad de los problemas ambientales, por una parte (no grave a muy grave), y, por otra, llevar a cabo una estimación del nivel de preocupación que los participantes manifestaran al contestar los ítems (no preocupación a muy preocupado). En ambos casos, tanto la puntación para las problemáticas como la preocupación manifestada por los participantes fue obtenida mediante el promedio de las calificaciones dadas en cada ítem. Para la categorización cualitativa se utilizaron las medidas de posición de los cuartiles.

Los datos obtenidos en las encuestas se subieron en una base de datos, elaborada en una hoja de cálculo, mediante la cual fue posible desarrollar el análisis descriptivo. Además, se utilizó el Índice de Jaccard (CantúMartínez, 2019) para medir la similitud entre las valoraciones expresadas de mujeres y varones sobre los problemas ambientales. Para establecer el contraste entre estos, se llevó a cabo la prueba de Z para dos proporciones, con un 95\% de confiabilidad (Daniel, 2006).

\section{Resultados}

\section{Descriptivos}

El total de estudiantes que participaron de las encuestas fue de 65 , de los cuales el $41,5 \%$ ( $\mathrm{f}=27$ ) fueron mujeres y el 58,4\% ( $\mathrm{f}=38)$ fueron varones. El promedio de edad de la población encuestada fue de 19,3 años $( \pm 1,1)$. Las mujeres tuvieron una edad media de 19,3 años ( $\pm 1,2$ años), mientras que los varones promediaron una edad de 19,4 años ( \pm 1,1años).

\section{Población total}

En relación con la preocupación mostrada por los alumnos universitarios, el $57 \%$ ( $\mathrm{f}=37$ ) manifestaron estar preocupados y el $43 \%(\mathrm{f}=28)$ poco preocupados por las problemáticas ambientales que se le plantearon en el ejercicio (tabla 1). Por una parte, con relación a la gravedad de los problemas esbozados, el $50 \%$ de estos $(\mathrm{f}=8)$ fueron contemplados como graves, entre los que encontramos en orden jerárquico las industrias y las pedreras $(2,04)$, el tráfico $(1,74)$, el ruido $(1,7)$, el poco interés por la conservación $(1,68)$, los residuos industriales $(1,66)$, la calidad de agua $(1,66)$, la contaminación ambiental $(1,58)$ y las aguas residuales $(1,54)$. Por otra parte, el $50 \%$ restante $(\mathrm{f}=8)$, se consideraron como poco graves la falta de espacios verdes $(1,48)$, los incendios forestales $(1,48)$, la destrucción de la naturaleza $(1,38)$, el incremento de la población $(1,34)$, los residuos urbanos (1,34), el despilfarro de energía (1,32), el incremento de la urbanización $(1,3)$ y, finalmente, la contaminación de los ríos $(1,26)$. 
TABLA 1.

Preocupación mostrada y problemáticas valoradas por los estudiantes universitarios que participaron en la investigación

\begin{tabular}{|c|c|c|c|c|}
\hline \multicolumn{5}{|c|}{ Preocupación } \\
\hline \multirow{2}{*}{ Categoría } & \multicolumn{2}{|c|}{ Preocupados } & \multicolumn{2}{|c|}{ Poco preocupados } \\
\hline & F & $\%$ & F & $\%$ \\
\hline Población total & 37 & 57 & 28 & 43 \\
\hline Mujeres & 20 & 74 & 7 & 26 \\
\hline Varones & 17 & 45 & 21 & 55 \\
\hline \multicolumn{5}{|c|}{ Problemáticas valoradas } \\
\hline \multirow[t]{2}{*}{ Categoría } & \multicolumn{2}{|c|}{$\begin{array}{c}\text { Problemas } \\
\text { graves }\end{array}$} & \multicolumn{2}{|c|}{$\begin{array}{c}\text { Problemas poco } \\
\text { graves }\end{array}$} \\
\hline & $\mathrm{F}$ & $\%$ & $\mathbf{F}$ & $\%$ \\
\hline Población total & 8 & 50 & 8 & 50 \\
\hline Mujeres & 10 & 62,5 & 6 & 37,5 \\
\hline Varones & 9 & 56,3 & 7 & 43,8 \\
\hline
\end{tabular}

\section{Mujeres}

En cuanto a la preocupación expresada por las estudiantes universitarias, el $74 \%(\mathrm{f}=20)$ declararon estar preocupadas y el $26 \%(\mathrm{f}=7)$ poco preocupadas por las problemáticas ambientales que se les plantearon. Así mismo, en lo relativo a las eventualidades ambientales evaluadas, las mujeres consideran que el $62,5 \%$ de estas $(\mathrm{f}=10)$ son graves, como lo son: los residuos industriales $(1,91)$, las industrias y pedreras $(1,82)$, el poco interés en la conservación $(1,82)$, el ruido $(1,77)$, la contaminación ambiental $(1,73)$, la calidad del agua $(1,68)$, el incremento de la población $(1,64)$, las aguas residuales $(1,59)$, el tráfico $(1,59)$ y los incendios forestales $(1,50)$. Mientras que el $37,5 \%(\mathrm{f}=6)$ fueron estimadas como poco graves: la falta de espacios verdes $(1,45)$, los residuos urbanos $(1,41)$, la contaminación de los ríos $(1,23)$, el despilfarro de energía $(1,23)$, la destrucción de la naturaleza $(1,18)$ y el incremento de la urbanización $(1,00)$.

\section{Varones}

Con respecto a la preocupación demostrada por los varones universitarios, el $45 \%$ de ellos $(\mathrm{f}=17)$ indicó estar preocupado, mientras que el 55\% $(\mathrm{f}=21)$ señaló estar poco preocupado. Con relación a las problemáticas ambientales propuestas en el estudio, los varones apuntaron que el $56,3 \%$ de estas $(f=9)$ son consideradas como graves; estas serían: las industrias y pedreras $(2,00)$, el tráfico $(1,86)$, el ruido $(1,64)$, la calidad de agua $(1,64)$, el poco interés en la conservación $(1,57)$, el incremento en la urbanización $(1,54)$, la destrucción de la naturaleza $(1,54)$, la falta de áreas verdes $(1,50)$ y las aguas residuales $(1,50)$. Mientras tanto, el $43,8 \%$ de las problemáticas $(\mathrm{f}=7)$ consideradas poco graves son: la contaminación ambiental $(1,46)$, los incendios forestales $(1,46)$, los residuos industriales $(1,46)$, el despilfarro de energía $(1,39)$, los residuos urbanos $(1,29)$, la contaminación de los ríos $(1,29)$ y, finalmente, el incremento de la población $(1,11)$.

\section{Mujeres vs. varones}

Alllevar a cabo el estudio de similitud y comparar entre las respuestas obtenidas, en relación con la gravedad de los eventos ambientales, encontramos que entre la población en general y las mujeres subsiste una similitud del $78 \%$, mientras que, con respecto a los varones, la similitud fue del $52,3 \%$. Al comparar los resultados obtenidos 
de las mujeres con los de los varones, hallamos una similitud entre estos del 39,1\% (tabla 2). Además, se concluyó que las mujeres demuestran una mayor preocupación que los varones $(\mathrm{p}<0,05)$.

TABLA 2.

Similitud entre las respuestas de la población total y los géneros participantes en la investigación

\begin{tabular}{lrrr}
\hline Grupo & Población total & Mujeres & Hombres \\
\hline Población total & & $78 \%$ & $52,3 \%$ \\
\hline Mujeres & $78 \%$ & & $39,1 \%$ \\
\hline Varones & $52,3 \%$ & $39,1 \%$ & \\
\hline
\end{tabular}

Fuente: elaboración propia

\section{Discusión}

El interés de los alumnos universitarios del área de las ciencias biológicas por las problemáticas ambientales planteadas, según los resultados obtenidos, es alta, ya que todos, tanto mujeres como varones, manifiestan estar preocupados por las evidentes problemáticas que subsisten en su entorno, lo cual coincide con la indagación realizada por Pavez-Soto et al. (2016), en Santiago de Chile.

Cabe mencionar que, en el caso del estudio presente, los estudiantes que participaron habían cursado durante su preparación académica una materia relativa al ámbito de la ecología y la biodiversidad, durante el segundo semestre de la carrera. Esta clase tuvo entre algunos de sus objetivos el uso y aprovechamiento responsable de los recursos naturales, como también el de los servicios ecosistémicos, lo que pretendía, mediante la formación académica, propiciar una conciencia ambiental (Universidad Autónoma de Nuevo León/Dirección de Estudios de Licenciatura [UANL/DEL], 2017; Universidad Autónoma de Nuevo León/ Facultad de Ciencias Biológicas [UANL/FCB], 2017; Cantú-Martínez, 2018). En este sentido, Antonio Gomera (2008) comenta que el

estudiante universitario se encuentra en una fase educativa clave en todos los ámbitos, incluido el ambiental. Aún es permeable a la adquisición de actitudes y aptitudes proambientales, probablemente en menor grado que en la etapa de Educación Primaria o Secundaria, pero con seguridad más que en la fase siguiente donde, como egresado, entrará en un mundo laboral que pone en juego muchos más condicionantes en la toma de decisiones que el puramente ambiental. Con todo ello, esta frontera en la que se ubica el universitario, entre la formación y el trabajo, puede suponer un prometedor nicho de adquisición de valores y conductas orientadas al desarrollo sostenible. (p. 6)

Así mismo, Benegas y Marcén (1995) indican que el contexto escolar puede ser concebido como un espacio de aprendizaje y desarrollo de actividades que tengan vinculación con el marco social, es decir, que puede desarrollar ciertos comportamientos tanto colectivos como individuales en todos los actores sociales; estas actividades conllevan, además, el propósito de inculcar determinados valores sociales que posteriormente se ostenten por los educandos en actitudes provechosas y adecuadas para el medio social en el que conviven. Aunado a lo anterior, comenta Cantú-Martínez (2014) que las instituciones educativas se erigen como el sitio donde no solo se comparte el conocimiento humano, sino que adicionalmente es el lugar en el cual las cuestiones del saber humano - científico, social y el de carácter práctico- se amalgaman en un solo.

Por lo tanto, los resultados obtenidos del ejercicio dan cuenta de una sensibilización de los participantes hacia los aspectos ambientales, producto del marco contextual social en el que se desenvuelven y que pondera sus posturas, todo mediante un proceso dialéctico entre la evidencia teórica, obtenida en su preparación académica, y el marco de la experiencia vivida (Durand, 2008; Casa et al., 2019). Además, conforme a lo que proponen Aguilar-Cucurachi et al. (2017), la conceptualización del entorno socioambiental está estrechamente vinculado con el entorno natural, en donde convergen los referentes tanto social como ecológico. Y, por consiguiente, "las actitudes y las creencias no pueden excluirse ni siquiera desde el acercamiento práctico, porque es práctico tomar en cuenta las emociones humanas en cualquier cálculo ambiental" (Yi-Fu, 2007, p. 10). 
Con respecto a la variable del género, Emakunde-Instituto Vasco de la Mujer (2014) pronuncia que la mujer cuenta con una percepción distinta a la del varón en el ámbito de las preocupaciones y aflicciones ambientales. Esto, esencialmente, se demuestra porque la mujer tiende a ser más participativa en los programas de conservación ambiental que el hombre. Inclusive, el Instituto apunta que las

mujeres contaminan menos que los hombres y sus actividades tienen un menor impacto sobre el deterioro del medio ambiente y del clima. Es decir, los hombres tienen comportamientos más destructivos del medio ambiente o lo que es lo mismo, dejan más huella ecológica sobre el planeta que las mujeres. (p. 4)

Lo anterior fue documentado con antelación por Davidson y Freudenburg (1996) al plantear que las mujeres demostraban mayores niveles de preocupación que los varones en temas relativos al ambiente y el uso de tecnología supeditada a la energía nuclear. Recientemente, en el trabajo de investigación llevado a cabo por Oliver et al., en el 2005, señalan que las mujeres cuentan con una mayor preocupación ante los problemas ambientales con respecto a la demostrada por los varones. Este supuesto se cumple también en nuestro estudio ( $74 \%$ vs. $45 \%$ ) y, a la vez, es estadísticamente significativo, pues cuenta con un valor de $\mathrm{p}<0,05$. Además, la similitud existente entre las opiniones de las mujeres y los varones fue solo del 39,1\%, lo que revela una forma distinta de juzgar las problemáticas ambientales, según nuestro estudio. Este aspecto también es corroborado por Mohai (1997), al concluir que las mujeres ostentan una mayor preocupación que los varones, particularmente, por las eventualidades ambientales, tanto localmente como de manera global.

Hace poco, McCright (2010) reportó que las mujeres estadounidenses muestran más preocupación que los varones, en un estudio sobre los efectos del cambio climático. Como también lo indica recientemente Calixto (2019) en su estudio sobre las actitudes de los jóvenes universitarios mexicanos hacia el cambio climático. Del mismo modo, Enrique-Mirón et al. (2012) comentan que, desde la perspectiva de género, habría que esperar una mayor sensibilidad de las mujeres con respecto a los eventos ambientales debido al binomio que se estructura entre la naturaleza y la mujer, y por el hecho de erigir la vida. Se concluye, entonces, que mujeres y varones interaccionan de modo disímil con el ambiente, ya sea en el ámbito natural o construido, como el urbano.

En relación con las implicaciones ambientales, sobresale de manera especial el problema que implican las industrias y las pedreras en el ANM. En particular, las industrias y las pedreras puntean en los resultados tanto en la evaluación de la población de estudio como entre las mujeres y los varones. Esto es, esencialmente, producto de que en los últimos años hayan existido muchos problemas ambientales debido al funcionamiento de estas. Daniel Anguiano (2017) señala en un reporte que el experto en temas de calidad de aire Gerardo Mejía atribuye el 30\% de las afectaciones del aire a los procesos industriales e indica que la problemática originada por la explotación de caliza contribuye en gran medida a la contaminación del ambiente en las áreas cercanas a los sitios de la explotación. Lo anterior ha llevado a que el ANM sea considerado por la Organización Mundial de Salud, en un estudio realizado en 1600 ciudades de 91 países, como la urbe que tiene la peor calidad del aire (Cantú-Martínez, 2015).

Adicionalmente, resultados como los obtenidos en este estudio contribuyen a la detección de las eventualidades ambientales percibidas por los estudiantes universitarios, lo que permite observar la diferencia existente entre las opiniones, planteada desde la perspectiva de género. En general, los hallazgos de esta indagación expresan que los alumnos universitarios se conciben como sujetos conocedores y preocupados ante las contingencias ambientales. El estudio también demuestra la experiencia aplicada del programa de educación para la sustentabilidad en el ámbito universitario, propuesto por la Unesco (2016), en el que la UANL estableció que todos los programas educativos ofrecidos impartieran valores y principios de sustentabilidad, con la finalidad de promover el pensamiento crítico en sus estudiantes para la comprensión y la resolución de los problemas sociales complejos y de los dilemas ambientales. Aún hoy se da continuidad a la Agenda 2030, a través de los Objetivos de Desarrollo Sostenible, específicamente, en el objetivo 4 y la meta 4,3, que plantean la educación para la sustentabilidad (Naciones Unidas, 2018). 
Como corolario, podemos también distinguir la imperiosa necesidad de seguir fortaleciendo los programas académicos que sobre sustentabilidad se imparten en la UANL, con el propósito de contribuir considerablemente a la formación de los estudiantes universitarios de manera preventiva, para posteriormente incidir en la labor profesional. Lo anterior conlleva también a el cumplimiento de las responsabilidades que atañen a la sociedad, esencialmente, después de haber adquirido un conocimiento tecnocientífico. Esto último, va de acuerdo con lo expresado por Simões et al. (2019), quienes señalan lacónicamente que el

único actor del ambiente que tiene la posibilidad de orientar sus acciones voluntariamente es el ser humano, por lo tanto, en él se centra la responsabilidad de promover un cambio cultural ligado a una nueva perspectiva ético-ambiental de la humanidad, que puede ser, la única salida a esta crisis. (p. 26)

\section{Referencias}

Aguilar-Cucurachi, M. S., Merçon, J., y Silva-Rivera, E. (2017). Aportaciones de las percepciones socio-ecológicas a la educación ambiental. Entreciencias, 5(15), 95-110. http://revistas.unam.mx/index.php/entreciencias/article/v iew/62581/55103

Álvarez, P., y Vega, P. (2009). Actitudes ambientales y conductas sostenibles. Implicaciones para la educación ambiental. Revista de Psicodidáctica, 14(2), 245-260. https://www.redalyc.org/pdf/175/17512724006.pdf

Anguiano, D. (2017, 10 de abril). Pedreras, ¿mayores culpables en la contaminación? Diario el Financiero. https://ww w.elfinanciero.com.mx/monterrey/especial-pedreras-mayores-culpables-en-la-contaminacion

Arias, C. A. (2006). Enfoques teóricos sobre la percepción que tienen las personas. Horizontes Pedagógicos, 8(19), 9-22. https://revistas.ibero.edu.co/index.php/rhpedagogicos/article/view/590

Barthey, S. H. (1982). Principios de percepción. Editorial Trillas.

Benegas, J., y Marcén, C. (1995). La educación ambiental como desencadenante del cambio de actitudes ambientales. Revista Complutense de Educación, 6(2), 11-28. https://revistas.ucm.es/index.php/RCED/article/view/RCED 9595220011A

Calixto, R. (2009). Representaciones sociales del medio ambiente. Universidad Pedagógica Nacional.

Calixto, R. (2019). Las representaciones sociales sobre el cambio climático de los estudiantes de pedagogía en México: un acercamiento desde la perspectiva de género. Educación, 28(54), 7-26. http://revistas.pucp.edu.pe/index.ph $\mathrm{p} /$ educacion/article/view/20777

Calixto, R., y Herrera, L. (2010). Estudio sobre las percepciones y la educación ambiental. Tiempo de Educar, 11(22), 227-249. https://www.redalyc.org/pdf/311/31121072004.pdf

Cantú-Martínez, P. C. (2014). Educación ambiental y la escuela como espacio educativo para la promoción de la sustentabilidad. Revista Electrónica Educare, 18(3), 39-52. https://www.scielo.sa.cr/pdf/ree/v18n3/a03v18n3. pdf

Cantú-Martínez, P. C. (2015). Escenario socioambiental del área metropolitana de Monterrey. Ciencia UANL, 18(72), 38-46. http://cienciauanl.uanl.mx/?p=3818

Cantú-Martínez, P. C. (2018). Alfabetización sobre cambio climático en estudiantes universitarios: un estudio exploratorio. En: J. Flores, I. L. Garza y A. I. Cuevas (eds.), Unidad y diversidad en educación, una visión multidisciplinaria (pp. 23-31). Universidad Autónoma de Nuevo León.

Cantú-Martínez, P. C. (2019). La apreciación semántica de la noción desafíos en bioética por alumnos universitarios del campo de las Ciencias Biológicas. Revista Iberoamericana de Bioética, 10, 1-11. https://revistas.comillas.edu /index.php/bioetica-revista-iberoamericana/article/view/11049

Carterette, E., y Friedman M. (1982). Manual de Percepción. Raíces Históricas y Filosóficas. Editorial Trillas.

Casa, M., Cusi, L., y Vilca, L. (2019). Percepciones sobre contaminación ambiental y actitudes en estudiantes universitarios. Revista Innova Educación, 1(3), 391-399. http://www.revistainnovaeducacion.com/index.php/r ie/article/view/128 
Clavijo, D. M., García, L. J., y Pulido, L. J. (2018). Relación entre la percepción ambiental y la actitud ambiental en un grupo de estudiantes de la Universidad Santo Tomás (tesis de Licenciatura). Universidad de Santo Tomás, Villavicencio, Colombia.

Daniel, W. (2006). Bioestadistica. Limusa y Wiley.

Davidson, D. J., y Freudenburg, W. R. (1996). Gender and environmental risk concerns. Environment and Behavior, 28, 302-339. https://doi.org/10.1177/0013916596283003

Durand, L. (2008). De las percepciones a las perspectivas ambientales. Una reflexión teórica sobre la antropología y la temática ambiental. Nueva Antropología, 21(68), 75-87. https://www.redalyc.org/pdf/159/15906805.pdf

Emakunde-Instituto Vasco de la Mujer (2014).La evaluación de impacto en función del género en el medio ambiente. España, Departamento de Medio Ambiente y Política Territorial del Gobierno Vasco.

Enrique-Mirón, C., Cabo-Hernández, J. M., y Mohamed-Abderraman, J. (2012, 31 de enero-3 de febrero). Sobre las diferencias de género en la percepción social del medio ambiente en estudiantes universitarios del campus de Melilla. Memoria del IX Congreso Iberoamericano de Ciencia, Tecnología y Género, Universidad de Sevilla (España).

Fernández, Y. (2008).¿Por qué estudiar las percepciones ambientales? Una revisión de la literatura mexicana con énfasis en Áreas Naturales Protegidas. Espiral, Estudios sobre Estado y Sociedad, 15(43), 179-202. http://www.scielo.o rg.mx/pdf/espiral/v15n43/v15n43a6.pdf

Gomera, A. (2008). La conciencia ambiental como herramienta para la educación ambiental: conclusiones y reflexiones de un estudio en el ámbito universitario. Argentina, Centro Nacional de Educación Ambiental. https://www.mi teco.gob.es/ca/ceneam/articulos-de-opinion/2008_11gomera1_tcm34-163624.pdf

Marín-Muñiz, J. L, Hernández, M. E., Silva, E., y Moreno-Casasola, P. (2016). Percepciones sobre servicios ambientales y pérdida de humedales arbóreos en la comunidad de Monte Gordo, Veracruz. Madera y Bosques, 22(1), 53-69. http://www.scielo.org.mx/pdf/mb/v22n1/1405-0471-mb-22-01-00053.pdf

McCright, A.M. (2010). The effects of gender on climate change knowledge and concern in the american public. Population and Environment, 32, 66-87. https://doi.org/10.1007/s11111-010-0113-1

Merleau-Ponty, M. (1993). Fenomenología de la percepción. Planeta-Agostini.

Mohai, P. (1997). Gender differences in the perception of most important environmental problems. Race, Gender and Class, 5, 153-169. https://www.jstor.org/stable/41674853?seq=1

Naciones Unidas. (2018). La Agenda 2030 y los Objetivos de Desarrollo Sostenible: una oportunidad para América Latina y el Caribe (LC/G.2681-P/Rev.3). Comisión Económica para América Latina y el Caribe (Cepal). https ://repositorio.cepal.org/bitstream/handle/11362/40155/24/S1801141_es.pdf

Oliver, M. F., Castells, M., Casero, A., y Morey, M. (2005). Actitudes y percepción del medio ambiente en la juventud española. España, Ministerio de Medio Ambiente de España.

Padilla, L. S., y Luna, A. M. (2003). Percepción y conocimiento ambiental en la costa de Quintana Roo: una caracterización a través de encuestas. Investigaciones Geográficas, 52, 99-116. http://www.scielo.org.mx/pdf/ige $\mathrm{o} / \mathrm{n} 52 / \mathrm{n} 52 \mathrm{a} 7 . \mathrm{pdf}$

Pavez-Soto, I., León-Valdebenito, C., y Triadú-Figueras, V. (2016). Jóvenes universitarios y medio ambiente en Chile: percepciones y comportamientos. Revista Latinoamericana de Ciencias Sociales, Niñez y Juventud, 14(2), 1435-1449. http://www.scielo.org.co/pdf/rlcs/v14n2/v14n2a38.pdf

Pidgeon, N. F. (1998), Risk assessment, risk values and the social science programme: why we do need risk perception research. Reliability Engineering \& System Safety, 59(1), 5-15. https://www.sciencedirect.com/science/article/ pii/S0951832097001142

Simões, A. S., Yanes, G., y Álvarez, M. (2019). Transversalidad de la educación ambiental para el desarrollo sostenible. Universidady Sociedad, 11(5), 25-32. http://rus.ucf.edu.cu/index.php/rus

Spinzi, C., Aquino, B., González, L., Wehrle, A., Scribano, R., y Jara, N. (2017). Educación ambiental y jóvenes: Influencia de las creencias y actitudes en comportamientos pro-ambientales en estudiantes de noveno grado, del departamento Central (2015)-Paraguay. Población y Desarrollo, 23(45), 16-24. https://dialnet.unirioja.es/serv let $/$ articulo?codigo $=6319455$ 
Unesco (2016). Formando el futuro que queremos. Decenio de las Naciones Unidas de la Educación para el Desarrollo Sostenible (2005-2014), Informe final-resumen. París, Sección de Educación para el Desarrollo Sostenible, División de Enseñanza, Aprendizaje y Contenido-Unesco.

Universidad Autónoma de Nuevo León/Dirección de Estudios de Licenciatura [UANL/DEL]. (2017). Programa analitico de la unidad de aprendizaje Ambiente y Sustentabilidad. San Nicolás de los Garza, Universidad Autónoma de Nuevo León/Dirección de Estudios de Licenciatura.

Universidad Autónoma de Nuevo León/Facultad de Ciencias Biológicas [UANL/FCB]. (2017). Programa analítico de la unidad de aprendizaje Ecología y Biodiversidad. San Nicolás de los Garza, Universidad Autónoma de Nuevo León/Facultad de Ciencias Biológicas.

Vargas, L. M. (1994). Sobre el concepto de percepción. Alteridades, 4(8), 47-53. https://www.redalyc.org/pdf/747/ 74711353004.pdf

Yi-Fu, T. (2007). Topofilia. Un estudio de las percepciones, actitudes y valores sobre el entorno. Melusina. https://www. melusina.com/rcs_gene/topofilia.pdf

\section{Notas}

* Artículo de investigación. Este artículo fue elaborado en el marco de la línea de investigación generación y aplicación del conocimiento, desarrollo humano, calidad de vida y sustentabilidad, que se desarrolla en el Cuerpo Académico 181-UANL de Ciencias Exactas y Desarrollo Humano, de la Facultad de Ciencias Biológicas de la Universidad Autónoma de Nuevo León. Se agradece al Consejo Nacional de Ciencia y Tecnología de México.

Licencia Creative Commons CC BY 4.0

Cómo citar este artículo: Cantú-Martínez, P. C. (2020). Preocupación y deterioro de la calidad ambiental. Apreciación de los estudiantes universitarios. Ambiente y Desarrollo, 24(46). https://doi.org/10.11144/Jav eriana.ayd24-46.pdca 